\title{
Timidina fosforilasa, lo bueno, lo malo y la oportunidad
}

\section{Thymidine phosphorylase, the good, the bad, and the opportunity}

LÓPEZ-HERNÁNDEZ, Eleazar†, VARGAS-HERNÁNDEZ, Genaro*, ÁLVAREZ-GARCÍA, Rocío y TOVAR-JIMÉNEZ, Xochitl

ID $1^{\text {er }}$ Autor: Eleazar, López-Hernández / ORC ID: 0000-0002-6466-1233, CVU Becario CONACYT: 782524

ID $1^{\text {er }}$ Coautor: Genaro, Vargas-Hernández / ORC ID: 000-0002-1931-2018, Researcher ID Thomson: Q-1235-2018, CVU CONACYT ID: 80183

ID $2^{\text {do }}$ Coautor: Rocío, Álvarez-García / ORC ID: 0000-0001-7573-3290, CVU CONACYT ID: 201058

ID $3^{\text {er }}$ Coautor: Xochitl, Tovar-Jiménez / ORC ID: 0000-0002-6626-6237, CVU CONACYT ID: 228873-SNI-1

DOI: $10.35429 /$ P.2020.4.28.38

E. López ${ }^{1}$, G. Vargas ${ }^{1,2}$, R. Álvarez, ${ }^{2}$, X. Tovar ${ }^{1,2}$

1. Programa Educativo de Doctorado en Ciencias en Biotecnología, Universidad Politécnica de Pachuca.

2. Programa Educativo de la Ingeniería en Biotecnología, Universidad Politécnica de Pachuca

*ghernan@upp.edu.mx

F. Trejo (Coord.). Ciencias Multidisciplinarias. Proceedings-CECORFAN-México, Pachuca, 2020. 


\begin{abstract}
Thymidine phosphorylase (TP) is an enzyme that belongs to the transferases that act on glycosyl groups, specifically on pentoses. TP participates in the metabolic pathway for the recovery or recycling of pyrimidines, for this, it catalyzes the phosphorylation of thymidine in ribose-1-phosphate and thymine, although the reaction is reversible, the main pathway is catabolic. Thymidine phosphorylase is a platelet-derived endothelial growth factor that facilitates angiogenesis, is overexpressed in cancer, favoring the metastasis process and has an inhibitory function on apoptosis. Due to the versatility of $\mathrm{TP}$, it is used in different areas, in biotechnology due to its activity for the modification of nucleosides and their analogues, for obtaining products for clinical use and as a therapeutic target for the development of enzyme inhibitors. In this work, we will describe the main structural and functional characteristics of TP, the lethal consequences of both its hyperactivity or overexpression and its absence or dysfunctionality, as well as the area of biotechnological opportunity provided by TP for drug development.
\end{abstract}

\title{
Thymidine phosphorylase, Cancer, Angiogenesis, Enzyme inhibitors, Non-nucleoside analogs
}

\section{Introducción}

La timidina fosforilasa (TP, por sus siglas en inglés), es una enzima que pertenece a las transferasas que actúan sobre grupos glicosil, específicamente sobre pentosas (Pugmire \& Ealick 2002). La TP participa en la ruta metabólica de recuperación o reciclaje de pirimidinas, para ello, cataliza la fosforilación de timidina en ribosa-1-fosfato y timina, aunque la reacción es reversible, la ruta principal es catabólica (Friedkin \& Roberts 1954). La timidina fosforilasa es un factor de crecimiento endotelial derivado de plaquetas que facilita la angiogénesis (Usuki et al., 1992), se sobreexpresa en cáncer favoreciendo el proceso de metástasis (Goto et al., 2012) y tiene una función inhibitoria sobre la apoptosis (Ikeda et al., 2008). Debido a la versatilidad de la TP, ésta se ocupa en diferentes áreas, en biotecnología por su actividad para la modificación de nucleósidos y sus análogos, para el desarrollo de productos de uso clínico y como blanco terapéutico para desarrollo de inhibidores de la enzima. En el presente trabajo se describen las principales características estructurales y funcionales de la TP, las consecuencias letales tanto de su hiperactividad o sobreexpresión como de la ausencia o disfuncionalidad de ésta, así como el área de oportunidad biotecnológica brindada por la TP para el desarrollo de fármacos.

\section{Antecedentes históricos de la Timidina Fosforilasa}

La TP se reportó por primera vez por (Deutsch \& Laser, 1929) como una nucleotidasa extraída de tejido animal, con la capacidad de liberación de ribosa a partir de timidina. Posteriormente Klein en 1935 en un trabajo similar, demostró la importancia de fosfato o arsenato para la activación de dicha catálisis. Por otro lado, Manson \& Lampen en 1951, sugirieron que el extracto de E. coli también tenía la actividad nucleosidasa y que además reconocía timina y uracilo como sustrato para la liberación de desoxirribosa 1 fosfato, aunque no identificaron el compuesto. Posteriormente, Friedkin \& Dewayne en 1954, purificaron esta enzima de tejido animal y comprobaron que se liberaba desoxirribosa 1 fosfato, compuesto que también sugirió Kalckar en 1945, pero utilizando nucleósidos con bases púricas. A la par, estudios de la uridín fosforilasa reportados, dieron pauta para que se dilucidara la primera estructura de la TP extraída de membranas de embrión (amnios y corion) (Kubilus, Lee, \& Baden, 1978). Esta enzima también se aisló de plaquetas y de neurofibroma y dado el efecto que esta tenía la nombraron factor de crecimiento endotelial derivado de plaquetas PD-ECGF (por sus siglas en ingles) y gliostatina (Asai et al., 1992). Sin embargo, al paso del tiempo se reportó que su secuencia es idéntica a la de la TP, esto abrió nuevas líneas de investigación y detectaron que la TP está presente en diversas células humanas como plaquetas, epitelios, células estromales, macrófagos, realizando funciones diferentes (Fox et al., 1995). En la menstruación se han encontrado niveles altos de la enzima en el endometrio, probablemente debido a la necesidad de generar nuevos vasos sanguíneos, ya que cuando termina el ciclo menstrual los niveles de TP se regulan notablemente (Zhang et al., 1997; Fujimoto et al., 1998; Sivridis et al., 2000). 


\section{Propiedades moleculares y bioquímicas de la TP}

El gen que codifica para la TP es deoA en E. coli y otros organismos del género enterobacter (Walter et al., 1990). En humanos se encuentra codificado en el cromosoma 22q13.33 (Ishikawa et al., 1989). Estos genes codifican para proteínas con estructura de homodímero (Walter et al., 1990) de 440 aminoácidos (aa) (47.21 KDa) y 482 aa $(49.96 \mathrm{KDa})$ respectivamente, su número de acceso al GenBank en el mismo orden es AYG20849.1 y ADP91533.1. Cada subunidad tiene un dominio mixto $\beta$ plegado y $\alpha$ hélice $(\alpha / \beta)$ el cual es largo en comparación al dominio pequeño $\alpha$ hélice que también posee esta proteína (Norman et al., 2004). Entre estos dos dominios se encuentra el sito de unión al sustrato de la enzima, de acuerdo con Panova et al., (2007) está conformado por los aminoácidos His85, Ser86, Gly88, Ser113, Thr123, Arg171 y Lys190. Además, el sitio activo se encuentra en una forma abierta cuando no está unido al sustrato, al unirse, se genera la conformación cerrada (Norman et al., 2004; El Omari et al., 2006). En la figura 1 se muestra la estructura de la TP, las flechas negras representan el sitio de unión al sustrato, cada subunidad tiene su propio sitio activo, el cual tiene una forma cerrada necesaria para que la enzima tenga actividad catalítica (Bronckaers et al., 2009).

Figura 1 Estructura tridimensional de la TPasa

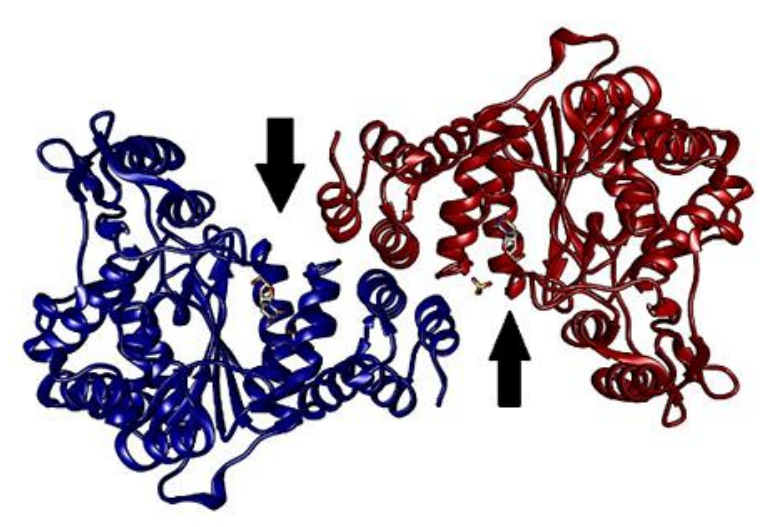

Cada color representa un monómero de la enzima, las flechas indican el sitio activo de ésta. Modelada con el programa Chimera 1.14 del formato publico $6 L U 7$

Con las cinéticas realizadas por Krenitsky y Tuttle en 1982 en TP de ratón y E. coli, así como las de Schwartz en 1971, con material de E. coli, se dilucidó el mecanismo de reacción. En este, el primer sustrato en unirse a la enzima (E) es el fosfato $(\mathrm{Pi})$, posteriormente ingresa la timidina (Td) formando el complejo $\mathrm{E}^{*} \mathrm{Pi}^{*} \mathrm{Td}$, con lo cual se cierra el sitio activo, dando lugar a los productos, que se liberan en un orden inverso al de entrada, primero la timina (T) seguido de la 2'desoxirribosa 1 fosfato (D1F). Por ello la nomenclatura que recibe el mecanismo es secuencial bi-bi ordenado.

Para la catálisis de la TP humana, los aminoácidos importantes son; His 116, Ser 217, Ile 214, Lys 115, Leu 148, Tyr 199 y Arg 202 (Miyadera et al., 1995; Mitsiki et al., 2009). Panova et al., 2007 remarcaron el papel que juega el grupo hidroxilo en la posición 3' ya que sin él no ocurre la transferencia de la base porque dicho grupo se une a la Thr 123 lo que ocasiona que la densidad electrónica se desplace a este sitio, dejando con una carga parcial positiva al $\mathrm{C} 1$ ', por lo tanto, es más susceptible a un ataque nucleofílico por parte del fosfato que está unido con la histidina 85 tal como se aprecia en la figura 2. También se observa que existe un estado de transición, en el cual están unidos la desoxirribosa, la timidina y el fosfato en la posición C1' (Edwards, 2006). Este estado es de suma importancia ya que basados en él y en los aminoácidos mencionados es como se han generado diversos inhibidores de transición, porque estos tienen una mayor afinidad al sitio activo (Mendieta et al., 2004). Por su parte Bronkaers et al., 2009, propusieron que el Asp 203 juega un papel crucial ya que permite la estabilización del loop, lo cual es indispensable en la catálisis. 
Figura 2 Mecanismo de acción y estado de transición de la TPasa

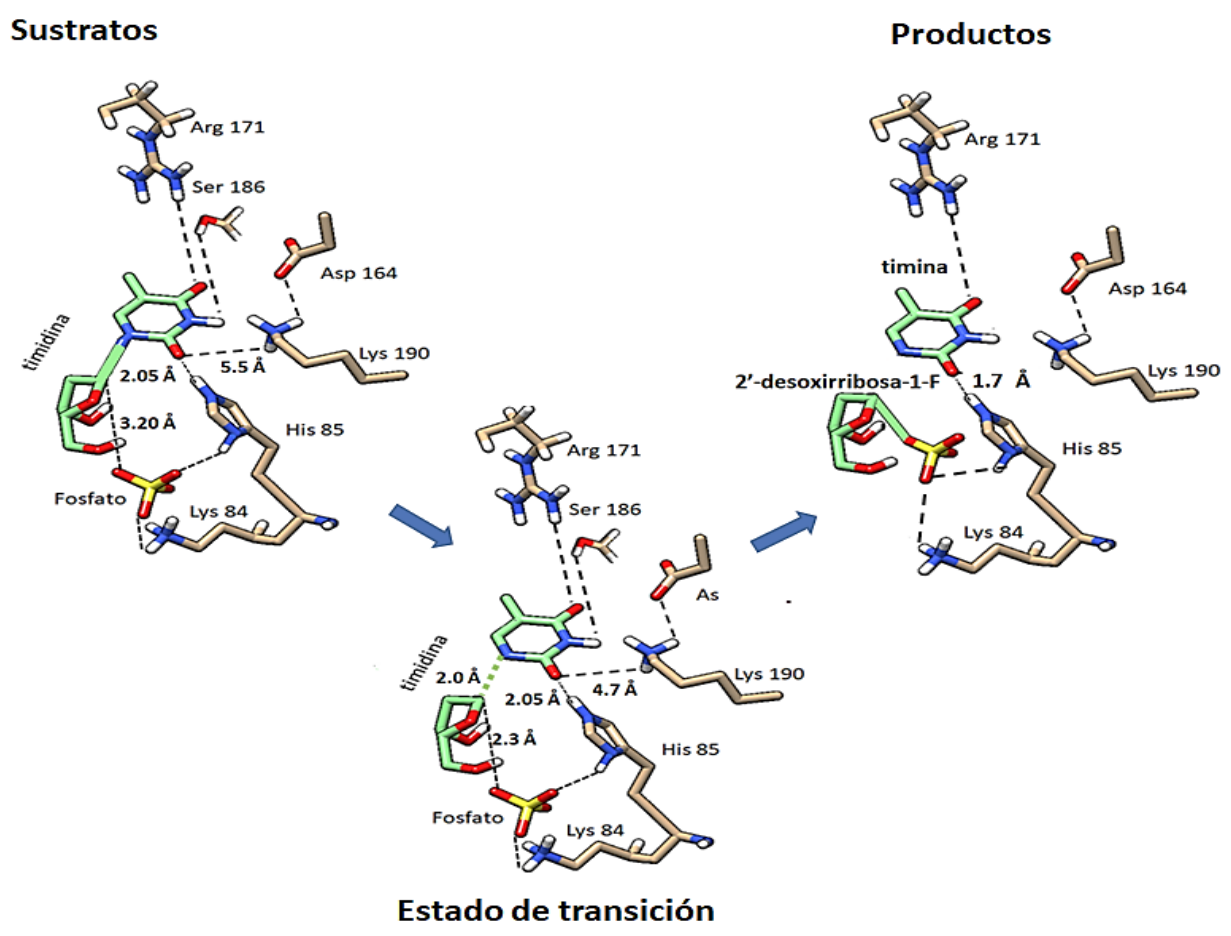

Las líneas punteadas representan enlaces no covalentes

Fuente: Tomada y modificada de Edwards et al. 2006

Cabe mencionar que estos aminoácidos están conservados en la mayoría de las enzimas obtenidas de diferentes organismos. En general toda la secuencia es 99\% idéntica en diversos microorganismos, los cuales incluyen Cronobacter, Edwardsiella, Yersenia, Serratia, Klebsiella, Hafnia, Ranhella, Proteus, Citrobacter, y E. coli solo por mencionar algunos. Cuando se analizaron las secuencias de peces, invertebrados o ranas se notó que comparten similitudes como los aminoácidos del sitio catalítico o unión a sustratos, así como la carencia de una sección rica en prolinas en el extremo $\mathrm{N}$ terminal, el cual si poseen los mamíferos y está relacionado con la homeostasis.

Para su actividad, la TP tiene una tolerancia de $\mathrm{pH}$ desde 5.3 hasta 10 dependiendo el microorganismo del que es extraído y las condiciones de reacción. Por ejemplo Finnis et al. (1993) reportaron un pH de 5.3 en TP humana (hTP), Zimmerman \& Seidenrberg (1964) encontraron un pH óptimo de 6 para la TP extraída de Cavia porcellus, Hommo sapiens, Rattus norvegicus y Mesocricetus auratus, Schwartz (1971) obtuvieron mayor actividad para la enzima extraída de E. coli (ecTP) a pH 7.1, y de Bruin et al. (2004) reportan el pH de 10 con la TP humana pero utilizaron esas condiciones para prevenir la degradación de la desoxirribosa 1 fosfato ante la exposición a altas temperaturas.

La temperatura en la que la TP presentó mayor actividad es de $37^{\circ} \mathrm{C}$ para aquellas enzimas extraídas de E. coli, Hommo sapiens y Salmonella typhimurium (Bakker, et al., 2010; Desgranges et al., 1981; Hoffee \& Blank, 1978; Mitsiki et al., 2009). No obstante, soporta temperaturas desde los 53-55 ${ }^{\circ} \mathrm{C}$ perdiendo hasta el 99\% actividad a los 10 min para la ecTP (Panova et al., 2008; Schwartz, 1971) mientras que la hTP pierde toda su actividad a los $65{ }^{\circ} \mathrm{C}$ (Desgranges et al., 1981). No obstante, la mayoría de las cinéticas realizadas en todo el mundo incluyendo proveedores de la enzima se realizan a $25^{\circ} \mathrm{C}$, tal y como lo propuso en un inicio Krenitsky y Tuttle (1982).

\section{El papel de la TP en la ruta de recuperación de bases pirimidínicas}

En esta ruta las bases pirimidínicas se reutilizan intercambiándolas con nucleósidos lo cual evita la síntesis de novo asegurando una fuente segura de monómeros para los procesos involucrados con el ADN, su reparación o replicación. La TP cataliza la conversión reversible de 2'-desoxi timidina en timidina libre y 2' desoxirribosa 1 fosfato (figura 3). Esta reacción puede terminar en la formación de la D1F si solo hay timina en el medio, pero si además se agrega alguna otra base, como citosina, la enzima tiene la capacidad de formar citidina o algún otro análogo de nucleósido (Liekens et al., 2007). 


\section{La TP como Factor de crecimiento y su implicación en cáncer}

En 1987, Miyazono et al., aislaron un factor a partir de plaquetas y lo nombraron PD-ECGF (por sus siglas en inglés) reportaron un fuerte efecto sobre el crecimiento endotelial. Años después se descubrió que su secuencia es idéntica a la TP. Como factor, esta proteína promueve el crecimiento de vasos sanguíneos de vascularización preexistente (Usuki et al., 1992), lo cual favorece a los tumores siendo esencial para su crecimiento y desarrollo (Goto et al., 2012). El mecanismo no está bien demostrado, pero se piensa que se debe a los subproductos ya que la timidina es catalizada en timina y D1F, la cual es desfosforilada a 2'desoxi-D-ribosa (2DDR) para ser liberada de la célula (Bijnsdorp et al., 2010). La 2DDR estimula la fosforilación de cinasas de adhesión en Tyr 397, la cual participa en la migración y muerte celular, además, la 2DDR activa las integrinas, lo que ocasiona una adhesión focal. Después, la molécula regula la proliferación celular y angiogénesis mediante la activación de p70/s6k (cinasa) de la ruta mTOR (Ricciuti et al., 2019). Por último, como la 2DDR es un azúcar, ésta provee de energía necesaria para la migración de endotelios, así como en la formación de pseudopodos (Bijnsdorp et al., 2010), por ello este monosacárido es considerado un blanco en el desarrollo de nuevos fármacos antitumorales. Algunos factores de angiogénesis están relacionados con el mismo efecto por parte de la TP, ya que si se suprime alguno de ellos se reduce la formación de vasos sanguíneos (Bijnsdorp et al., 2010). El factor de hipoxia inducible (HIF 2- $\alpha$ ) también se sobreexpresa en células cancerosas teniendo una correlación estadísticamente significativa con la expresión de la TP (Moreno et al., 2018). De hecho, esta es una de las razones por las que la TP se sobreexpresa en células cancerosas, por ejemplo, las de cáncer de mama (Ruckhäberle et al., 2010), de vejiga (Shimabukuro et al., 2005), cérvico uterino (Hasegawa et al., 2012), colorrectal (Sadahiro et al., 2012) o de esófago (Wang et al., 2012), ya que los tumores requieren muchos nutrientes, los cuales adquieren gracias a sus proliferados vasos sanguíneos. Esto indica que los nuevos vasos sanguíneos formados permiten una oxigenación mejor en el microambiente tumoral, lo que conlleva a una disminución de HIF 1- $\alpha$ junto con la regulación de HIF $2-\alpha$, que, a su vez, induce la activación de la TP y la ciclooxigenasa (COX-2) para mantener la angiogénesis (De Bruin et al., 2006).

Figura 3 Catálisis realizadas por la enzima Timidina fosforilasa

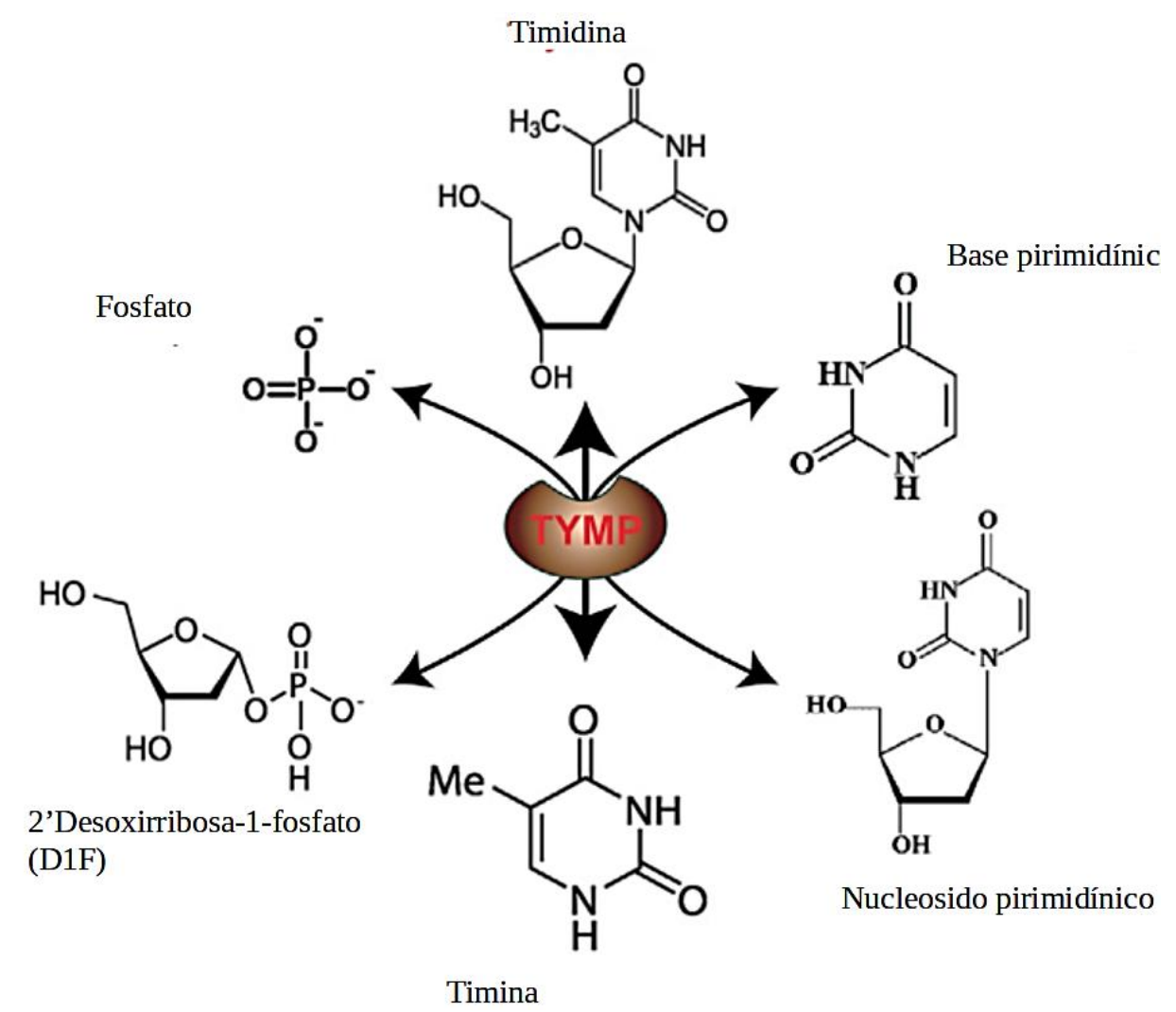

Fuente: Tomado y modificado de Li \& Yue, 2018. 
Además de lo anterior, la TP inhibe la apoptosis por 4 vías, una es la regulación de Bcl-2 y Bclxl, otra es impidiendo la liberación de citocromo c de las mitocondrias, también evita la fosforilación de p38 proteína cinasa activada por mitógeno (MAPK) y finalmente mediante la inactivación de las caspasas 9 y 3 (Ikeda et al., 2008; 2002). El proceso es el siguiente; bajo condiciones de hipoxia, 2DDR evita que se fosforile MAPK, por consiguiente, no activa a BAX impidiendo que se libere el citocromo c y la activación de caspasas. La TP también puede interferir con la liberación de citocromo c y la caspasa 3 independientemente de su actividad catalítica. Es capaz de aumentar la liberación de interleucina (IL)-10, la cual inhibe la respuesta antiinflamatoria de células dendríticas, así como de macrófagos (Li et al., 2008, Ikeda et al., 2008). La TP induce la expresión de metaloproteinasas vía activación de la ruta de AKT, que a su vez regula el activador de proteínas 1 (AP1), el cual participa en la transcripción de las metaloproteinasas, esto promueve la movilidad y metástasis de las células cancerosas (Ko et al., 2014). Por lo anterior la TP es un factor clave en el desarrollo de factores característicos del cáncer, de hecho, su sobreexpresión en algunos tipos de cáncer está relacionada con un mal pronóstico y al mismo tiempo, la TP también es clave para la determinación de la respuesta a algunos agentes terapéuticos.

\section{Implicación de la TP en otros trastornos}

Li et al. en 2014 demostraron que la TP tiene una función importante en la activación plaquetaria y promueve la trombosis, reportaron que la administración in vivo de KIN59 inhibió significativamente la trombosis de la arteria carótida inducida por $\mathrm{FeCl}_{3} \sin$ afectar la hemostasia. Por lo que se propone a la TP como blanco atractivo para enfermedades cardiovasculares ( $\mathrm{Li}, 2018)$. La TP también se ha reportado en la placa aterosclerótica, sin embargo, podría tener una función dual, se considera que contribuye al crecimiento y ruptura de la placa (Ignatescu et al., 1999;) sin embargo, también se reportó que la alta expresión de TYMP en la placa aterosclerótica en realidad puede atenuar la respuesta de la pared del vaso VSMC a la estimulación fisiopatológica (Handa et al., 2008).

Por otro lado, la pérdida de la función de la TP, causante de la encefalomiopatía mitocondrial neurogastrointestinal (enfermedad autosómica recesiva), induce a la acumulación de nucleósidos en plasma y tejidos, así como disfunción mitocondrial, neuropatía periférica que conduce a la muerte (Filosto et al., 2018), por lo que su diagnóstico temprano, así como la administración de TP podría conferir buenos resultados terapéuticos.

\section{El papel biotecnológico de la TP en la síntesis de análogos de nucleósidos}

Dada la función ya descrita de la TP, esta enzima se ha utilizado en la síntesis de moléculas similares en estructura a los nucleósidos naturales, es decir, análogos. Estos compuestos se caracterizan porque tienen alguna porción modificada o eliminada en la base o en el azúcar, lo cual impide que sean usados adecuadamente en la síntesis de ADN. Por ello mismo es que son empleados mayoritariamente en enfermedades en las que se requiere detener la replicación de material genético (Nakayama et al., 1980; Desgranges et al., 1983; Lapponi et al., 2016). A pesar de la enantio y regioselectividad de la TP, reconoce sustratos diferentes, principalmente a bases modificadas, aunque también a nucleótidos con modificaciones en el azúcar. Así, partiendo de un nucleósido, se pueden obtener diversos análogos como se ejemplifica en la tabla 1. No obstante el hidroxilo de la posición 3' no debe de eliminarse o la enzima pierde toda su actividad (Panova et al., 2007). Algunas de esas modificaciones se observan en la tabla 1, donde se encuentra el nucleósido donador y la base receptora del azúcar. De la misma se destacan algunas modificaciones como en el caso de la 2,3, desoxitimidina, que a pesar de que carece de 2 grupos hidroxilo aún se observa actividad. Todo lo contrario, a los reportado por Panova et al., en 2007, quienes al eliminar el $\mathrm{OH} 3$ ' o cambiarlo de posición espacial, observaron que se perdía toda la actividad e incluso inhibían a la enzima. Por otro lado, la 3'-amino-3'-desoxitimidina no es un sustrato para la enzima a un $\mathrm{pH}$ de 6.5 pero si lo es cuando el $\mathrm{pH}$ alcanza un valor cercano a 8 . Lo que se debe a que a pH 8 el grupo amino está desprotonado y no interfiere con la formación del puente de hidrógeno con el aa Thr 123, pero a pH 6.5 ambos están protonados lo que ocasiona una repulsión. En el caso del 2',3' didehidro 2',3', didesoxitimidina se observó actividad, aunque fue 100 veces menor a lo normal. Por otro lado, esta enzima se utilizó para la síntesis de ribavirina, excelente fármaco antiviral (Ding et al., 2010). En base a todo lo mencionado anteriormente, nuestro grupo de investigación está sintetizando fármacos y biofármacos que interactúen con esta enzima. 
Tabla 1 Sustratos reconocidos por la timidina fosforilasa

\begin{tabular}{|c|c|c|c|c|}
\hline $\begin{array}{c}\text { Donador de } \\
\text { desoxirribosa }\end{array}$ & $\begin{array}{c}\text { Aceptor de } \\
\text { desoxirribosa }\end{array}$ & Producto & $\begin{array}{c}\% \text { de } \\
\text { Conversión }\end{array}$ & Referencia \\
\hline Uridina & ---- & Uracilo + D1F & 100 & \multirow{5}{*}{$\begin{array}{l}\text { (Schinazi et al. } \\
\text { 1992) }\end{array}$} \\
\hline floururidina & ---- & 5-floururacilo + D1F & 99 & \\
\hline 2,3' didesoxiuridina & ---- & Uracilo + 2',3' di-D1F & 55.3 & \\
\hline 2,3 desoxitimidina & $\begin{array}{ll}--- \\
\end{array}$ & Timidina $+\mathrm{D} 1 \mathrm{~F}$ & 31.9 & \\
\hline 5'flour-D1F & Uracilo & 5'-flour-5'- desoxiuridina & $\mathrm{Nd}$ & \\
\hline 5'flour 5'D1F & 5-floururacilo & 5'-flour-5'- desoxifloururidina & $\mathrm{Nd}$ & \multirow{2}{*}{$\begin{array}{l}\text { (Panova et al., } \\
\text { 2007) }\end{array}$} \\
\hline 5'bromo 5' D1F & 5-bromouracilo & $\begin{array}{ll}\text { 5'-flour-5' } & \text { desoxi } \\
\text { bromouridina } & \\
\end{array}$ & $\mathrm{Nd}$ & \\
\hline Timidina & Adenina & Desoxiadenosina* & 87.4 & \multirow[t]{4}{*}{ (Ding et al., $\quad$ 2010) } \\
\hline Timidina & Diaminopurina & Desoxidiaminopurina* & 84.1 & \\
\hline Timidina & Hipoxantina & Desoxiinosina* & 52.7 & \\
\hline Timidina & Triazolcarboxi amina & Desoxi- rivavirina* & 79.5 & \\
\hline 2'desoxiuridina & -- & $\begin{array}{l}\mathrm{D} 1 \mathrm{~F}+ \\
\text { Uracilo }\end{array}$ & $\mathrm{Nd}$ & \multirow[t]{3}{*}{ (Serra et al., $\quad$ 2013) } \\
\hline 5' desoxiuridina & --- & $\begin{array}{lll}\text { 5'-desoxirribosa } & 1 & \text { fosfato } \\
(5 ' D 1 F)+ & & \\
\text { Uracilo } & & \end{array}$ & $\mathrm{Nd}$ & \\
\hline Uridina & --- & $\begin{array}{l}\text { Ribosa } 1 \text { fosfato }(\mathrm{R} 1 \mathrm{~F})+ \\
\text { Uracilo }\end{array}$ & $\mathrm{Nd}$ & \\
\hline 5'tiotimidina & --- & $\begin{array}{l}\text { Timina }+ \\
\text { 5'-tio-D1F }\end{array}$ & 39 & \multirow[t]{4}{*}{ (Hatano et al., 2008) } \\
\hline 3' tiotimidina & --- & $\begin{array}{l}\text { Timina }+ \\
\text { 3'-tio-D1F }\end{array}$ & 0.76 & \\
\hline 6-azatimidina & --- & 6-Azatimina + D1F & 5.4 & \\
\hline $\begin{array}{l}\text { 5' cloro } 5 \text { ' desoxi } \\
\text { timidina }\end{array}$ & --- & $\begin{array}{l}\text { Timina }+ \\
\text { 5'-cloro 5'-D1F }\end{array}$ & 24 & \\
\hline
\end{tabular}

\section{Conclusiones}

La versatilidad en las funciones de la TP la convierten en una enzima clave para el metabolismo celular y tanto su hiperactividad, sobreexpresión o disfuncionalidad conllevan al desarrollo de diversas patologías en las que se involucra a los ácidos nucleicos y, por ende, son el blanco perfecto para diferentes tratamientos, especialmente en terapias anticancerígenas. Por otro lado, la actividad de la TP en la modificación de nucleótidos y su baja especificidad las hace atractivas para su uso en la síntesis de nuevos análogos de nucleósidos, como fármacos potencialmente aplicables en diferentes patologías.

\section{Agradecimiento}

El autor López Hernández Eleazar agradece la beca Conacyt no. 728384 para sus estudios de doctorado

\section{Referencias}

Asai K., Hirano T., Kaneko S., Moriyama A., Nakanishi K., Isobe I., Eksioglu Y. Z. \& Kato T. (1992). A novel glial growth inhibitory factor, gliostatin, derived from neurofibroma. Journal of Neurochemistry, 59, 307-317

Bakker J. A., Schlesser P., Smeets H. J., Francois B. \& Bierau J. (2010). Biochemical abnormalities in a patient with thymidine phosphorylase deficiency with fatal outcome. Journal of Inherited Metabolic Disease, 33 (Suppl 3), S139-S143.

Bijnsdorp I. V., Vrijland K., Vroling L., Fukushima M. \& Peters G.J. (2010). Increased migration by stimulation of thymidine phosphorylase in endothelial cells of different origin. Nucleosides Nucleotides Nucleic Acids, 29(4-6), 482-487

Blank J. G. \& Hoffee P. A. (1975). Purification and properties of thymidine phosphorylase from Salmonella typhimurium. Archives of Biochemistry and Biophysics, 168, 259-265 
Bronckaers A., Aguado L., Negri A., Camarasa M. J., Balzarini J., Perez-Perez M. J., Gago F. \& Liekens S. (2009). Identification of aspartic acid-203 in human thymidine phosphorylase as an important residue for both catalysis and non-competitive inhibition by the small molecule "crystallization chaperone" 5'-O-tritylino- sine (KIN59). Biochemistry Pharmacology, 78, 231-40.

Brown N. S. \& Bicknell R. (1998). Thymidine phosphorylase, 2-deoxy- d-ribose and angiogenesis. Biochemical Journal, 334 (Pt 1), 1-8

de Bruin M., Temmink O. H., Hoekman K., Pinedo H. M. \& Peters G. J. (2006). Role of platelet derived endothelial cell growth factor/ thymidine phosphorylase in health and disease. Cancer Therapy, 4, 99-124

de Bruin M., van Capel T., Smid K., van der Born K., Fukushima M., Hoekman K., Pinedo H. M. \& Peters G. J. (2004). Role of platelet derived endothelial cell growth factor/thymidine phosphorylase in fluoropyrimidine sensitivity and potential role of deoxy- ribose-1-phosphate. Nucleosides Nucleotides Nucleic Acids, 23,1485-90.

Desgranges C., Razaka G., Rabaud M., Bricaud H., Balzarini J. \& De Clercq E. (1983). Phosphorolysis of (E)-5-(2-bromovinyl)-2'- deoxyuridine (BVDU) and other 5-substituted-2'-deoxyuridines by purified human thymidine phosphorylase and intact blood platelets. Biochemical Pharmacology, 32, 3583-90.

Desgranges C., Razaka G., Rabaud M. \& Bricaud H. (1981). Catabolism of thymidine in human blood platelets purification and properties of thymidine phosphorylase. Biochimica et Biophysica Acta, 654, 211-218. 39.

Deutsch W., \& Laser R. Z. (1929). Experimentelle Studien über den Nucleinstoffwechsel. XIX. Mitteilung. Zur Kenntnis der Nucleosidase. Verhalten einer Nucleosidase aus Rinderknochenmark zu einem Spaltprodukt der Thymusnucleinsäure. Physiological Chemistry, 186, 1.

Ding Q. B., Ou L., Wei D. Z., Wei X. K., Xu Y. M., \& Zhang C. Y. (2010). Enzymatic synthesis of nucleosides by nucleoside phosphorylase co-expressed in Escherichia coli. Journal of Zhejiang University Science B, 11(11), 880-888.

Edwards P.N. (2006). A kinetic, modeling and mechanistic re-analysis of thymidine phosphorylase and some related enzymes. Journal of Enzyme Inhibition and Medicinal Chemistry, 21(5), 483-499.

El Omari K., Bronckaers A, Liekens S., Perez-Perez M.J., Balzarini J., Stammers D.K. (2006). Structural basis for non-competitive product inhibition in human thymidine phosphorylase: implications for drug design. Biochemical Journal, 399, 199-204.

Filosto M., Cotti Piccinelli, S. Caria, F., Gallo Cassarino, S., Baldelli, E., Galvagni, A., Volonghi, I., Scarpelli, M., Padovani, A. (2018). Mitochondrial Neurogastrointestinal Encephalomyopathy (MNGIEMTDPS1). Journal of Clinical Medicine, 7, 389.

Finnis C., Dodsworth N., Pollitt C. E., Carr G. \& Sleep D. (1993). Thymidine phosphorylase activity of platelet-derived endothelial cell growth factor is responsible for endothelial cell mitogenicity. European. Journal Biochemistry, 212, 201-2 10

Fox S. B., Moghaddam A., Westwood M., Turley H., Bicknell R., Gatter K.C. \& Harris A. L. (1995). Platelet-derived endothelial cell growth factor/thymidine phosphorylase expression in normal tissues: an immunohistochemical study. Journal of Pathology. 176, $183-190$.

Friedkin M. \& Roberts D. (1954). The enzymatic synthesis of nucleosides. I. Thymidine phosphorylase in mammalian tissue. Journal of Biological Chemistry, 207(1), 245-256.

Fujimoto J., Ichigo S., Sakaguchi H., Hirose R. \& Tamaya T. (1998). Expression of platelet-derived endothelial cell growth factor and its mRNA in uterine endometrium during the menstrual cycle. Molecular Human Reproduction, 4, 509-13 
Gotanda T., Haraguchi M., Tachiwada T., Shinkura R., Koriyama C., Akiba S. ... Nakagawa M. (2006). Molecular basis for the involvement of thymidine phosphorylase in cancer invasion. International Journal of Molecular Medicine, 17(6), 1085-1091.

Goto T., Shinmura K., Yokomizo K., Sakuraba K., Kitamura Y., Shirahata A. ... Kenji H. (2012) Expression levels of thymidylate synthase, dihydropyrimidine dehydrogenase, and thymidine phosphorylase in patients with colorectal cancer. Anticancer Research, 32:1757-62.

Handa M., Li W., Morioka K., Takamori A., Yamada N. \& Ihaya A. (2008) Adventitial delivery of platelet derived endothelial cell growth factor gene prevented intimal hyperplasia of vein graft. Journal of Vascular Surgery, 48, 1566-74.

Hasegawa K., Okamoto H., Kawamura K., Kato R., Kobayashi Y., Sekiya T. \& Udagawa Y. (2012). The effect of chemotherapy or radiotherapy on thymidine phosphorylase and dihydropyrimidine dehydrogenase expression in cancer of the uterine cervix. European Journal of Obstetrics, Gynecology, and Reproductive Biology, 163(1), 67-70.

Hemalatha T., Tiwari M., Balachandran C., Manohar B.M., Puvanakrishnan R. (2009). Platelet-derived endothelial cell growth factor mediates angiogenesis and antiapoptosis in rat aortic endo- thelial cells. Biochemistry of Cellular Biology, 87(6), 883-893

Ignatescu M. C., Gharehbaghi-Schnell E., Hassan A., Rezaie-Majd S., Korschineck I., Schleef R. R., Glogar H. D., \& Lang I. M (1999). Expression of the angiogenic protein, platelet-derived endothelial cell growth factor, in coronary atherosclerotic plaques: In vivo correlation of lesional microvessel density and constrictive vascular remodeling. Arteriosclerosis, Thrombosis, and Vascular Biology, 19(10), 2340-7.

Ikeda R., Furukawa T., Kitazono M., Ishitsuka K., Okumura H., Tani A. .. Akiyama S. (2002). Molecular basis for the inhibition of hypoxia- induced apoptosis by 2-deoxy-D-ribose. Biochemical and Biophysical Research Communications, 291(4), 806-812

Ikeda R., Tajitsu Y., Iwashita K., Che X.F., Yoshida K., Ushiyama M. .. Yamada K. (2008). Thymidine phosphorylase inhibits the expression of proapoptotic protein BNIP3. Biochemical and Biophysical Research Communications, 370(2), 220-224.

Ishikawa F., Miyazono K., Hellman U., Drexler H., Wernstedt C., Hagiwara K., Usuki K., Takaku F., Risau W. \& Heldin C.H. (1989) Identification of angiogenic activity and the cloning and expression of platelet-derived endothelial cell growth factor. Nature, 338, 557- 562.

Kalckar H. M. (1945) The enzymatic synthesis of a nucleoside. Journal of Biological Chemistry, 158(3) 723-724

Klein W., Z. (1935). Experimentelle Studien über den Nucleinstoffwechsel. Über Nucleosidase Physiology Chemistry, 231: 125

Ko J. C., Chiu H. C., Syu J. J., Jian Y. J., Chen C. Y., Jian Y. T., Huang Y. J., Wo T. Y. \& Lin, Y. W. (2014). Tamoxifen enhances erlotinib-induced cytotoxicity through down-regulating AKT-mediated thymidine phosphorylase expression in human non- small-cell lung cancer cells. Biochemical Pharmacology. 88(1), 119-27.

Krenitsky, T. A., Tuttle, J. V. (1982). Correlation of substrate-stabilization patterns with proposed mechanisms for three nucleoside phosphorylases. Biochimica et biophysica Acta, 703 (2), 247-249.

Kubilus J., Lee L.D., Baden H.P. (1978). Purification of thymidine phosphorylase from human amniochorion. Biochimica et biophysica Acta, 527(1), 221-228.

Lapponi M. J., Rivero C. W., Zinnia M. A., Britosa C. N., Trelles J. A. (2016). New developments in nucleoside analogues biosynthesis: A review. Journal of Molecular Catalysis B: Enzymatic, 133, 218233 
Li W., Gigante A., Perez-Perez M.J., et al., (2014). Thymidine phosphorylase participates in platelet signaling and promotes thrombosis. Circylation Research, 115(12), 997-1006.

Li W., Tanaka K., Morioka K., Takamori A., Handa M., Yamada N. \& Ihaya, A. (2008). Long-term effect of gene therapy for chronic ischemic myocardium using platelet-derived endothelial cell growth factor in dogs. The Journal of Gene Medicine, 10, 412-20

Li W., Yue H. (2018). Thymidine phosphorylase: A potential new target for treating cardiovascular disease. Trends in Cardiovascular Medicine, 28(3),157-171.

Liekens S., Bronckaers A., Perez-Perez M.J. \& Balzarini J. (2007). Targeting platelet-derived endothelial cell growth factor/ thymidine phosphorylase for cancer therapy. Biochemical Pharmacology. 74(11), 1555-67.

Manson L. A. \& Lampen L.O. (1951). The metabolism of desoxyribose nucleosides in Escherichia coli. Journal of Biological Chemistry, 193, 539-547

Mendieta J., Martín-Santamaría S., Priego E. M., Balzarini J, Camarasa M. J., Pérez-Pérez M. J., \& Gago F. (2004). Role of Histidine-85 in the Catalytic Mechanism of Thymidine Phosphorylase as Assessed by Targeted Molecular Dynamics Simulations and Quantum Mechanical Calculations. Biochemistry, 43(2), 405-414

Mitsiki E., Papageorgiou A. C, Iyer S., Thiyagarajan N., Prior S. H., Sleep D., Finnis C. \& Acharya K. R. (2009). Structures of native human thymidine phosphorylase and in complex with 5-iodour- acil. Biochemical and Biophysical Research Communications, 386, 666-70.

Miyadera K., Sumizawa T., Haraguchi M., Yoshida H., Konstanty W., Yamada Y. \& Akiyama S. (1995) Role of thymidine phosphorylase activity in the angiogenic effect of platelet derived endothelial cell growth factor/thymidine phosphorylase. Cancer Research, 55(8), 1687-90.

Miyazono K., Okabe T., Urabe A., Takaku F \& Heldin C. H. (1987). Purification and properties of an endothelial cell growth factor from human platelets. Journal of Biological Chemistry, 262, 4098-4103.

Moreno R. E., Yaromina, A., Houben, R., Groot, A. J., Dubois, L., \& Vooijs, M. (2018). Prognostic Role of Hypoxia-Inducible Factor-2 $\alpha$ Tumor Cell Expression in Cancer Patients: A Meta-Analysis. Frontiers in Oncology, 8, 224.

Nakayama C., Wataya Y., Meyer R. B. Jr, Santi D. V., Saneyoshi M. \& Ueda T. (1980). Thymidine phosphorylase. Substrate specificity for 5-substituted 2'-deoxyuridines. Journal of Medicinal Chemistry. 23(8), 962-4.

Norman R. A., Barry S. T., Bate M., Breed J., Colls J. G., Ernill R. J. ... Pauptit R. A. (2004). Crystal structure of human thymidine phosphorylase in complex with a small molecule inhibitor. Structure. 12(1), 75-84.

Panova I. G., Sharova N. P., Dmitrieva S. B., Poltavtseva R. A., Sukhikh G. T. \& Tatikolov A. S. (2007). Substrate specificity of Escherichia Coli thymidine phosphorilase. Biochemistry (Moscow), 72(1), 21-8.

Pugmire M. J. \& Ealick S. E. (2002). Structural analyses reveal two distinct families of nucleoside phosphorylases. Biochemical Journal, 361(1), 1-25.

Pula G., Mayr U., Evans C., Prokopi M., Vara D. S., Yin X., Astroulakis Z., Xiao Q., Hill J., Xu Q. \& Mayr M. (2009) Proteomics identifies thymidine phosphorylase as a key regulator of the angiogenic potential of colony-forming units and endothelial progenitor cell cultures. Circulatory Research, 104(1), 32-40. 
Raymond F. Schinazi, A. P., Jean-Pierre S. (1992). Substrate specificity of Escherichia coli thymidine phosphorylase for pyrimidine nucleosides with anti-human immunodeficiency virus activity. Biochemical Pharmacology, 44(2). 199-204.

Ricciuti, B., Leonardi, G. C., \& Brambilla, M. (2019). Emerging Biomarkers in the Era of Personalized Cancer Medicine. Disease Markers, 5907238.

Ruckhäberle E., Karn T., Engels K., Turley H., Hanker L., Müller V. ... Rody A. (2010). Prognostic impact of thymidine phosphorylase expression in breast cancer-comparison of microarray and immunohistochemical data. European Journal of Cancer, 46(3), 549-557.

Sadahiro S., Suzuki T., Tanaka A., Okada K., Nagase H., Uchida J. (2012) Association of right-sided tumors with high thymidine phosphorylase gene expression levels and the response to oral uracil and tegafur/leucovorin chemotherapy among patients with colorectal cancer. Cancer Chemotherapy Pharmacology. 70(2):285-291.

Schwartz M. (1971). Thymidine phosphorylase from Escherichia coli. Properties and kinetics. European Journal of Biochemistry, 21:191-8.

Serra I., Teodora Bavaro T., Cecchini D. A., Daly S., Albertini A. M., Terreni M., Ubiali D. (2013). A comparison between immobilized pyrimidine nucleoside phosphorylase from Bacillus subtilis and thymidine phosphorylase from Escherichia coli in the synthesis of 5-substituted pyrimidine 2'deoxyribonucleosides. Journal of Molecular Catalysis B: Enzymatic, 95:16-22.

Shimabukuro T., Matsuyama H., Baba Y., Jojima K., Suyama K., Aoki A., Suga A., Yamamoto N. \& Naito, K (2005). Expression of thymidine phosphorylase in human superficial bladder cancer. International Journal of Urology, 12(1), 29-34.

Sivridis E., Giatromanolaki A., Koukourakis M. I., Bicknell R., Harris A. L. \& Gatter K. C. (2000). Thymidine phosphorylase expression in normal and hyperplastic endometrium. Journal of Clinical Pathology, 53(9), 704-708.

Usuki, K., Saras, J., Waltenberger, J., Miyazono, K., Pierce, G., Thomason, A. \& Heldin, C. H. (1992). Platelet-derived endothelial cell growth factor has thymidine phosphorylase activity. Biochemical and Biophysical Research Communications, 184(3),1311-1316.

Walter, M. R., Cook, W. J., Cole, L. B., Short, S. A., Koszalka, G. W., Krenitsky, T. A. \& Ealick E. (1990). Three-dimensional structure of thymidine phosphorylase from Escherichia coli at $2.8 \mathrm{~A}$ resolution. The Journal of Biological Chemistry, 265(23), 14016-14022.

Wang L., Huang X., Chen Y., Jin X., Li Q. \& Yi T.N. (2012). Prognostic value of TP/PD-ECGF and thrombocytosis in gastric carcinoma. European Journal of Surgical Oncology, 38(7), 568-573.

Zhang L., Mackenzie I. Z., Rees M. C. \& Bicknell R. (1997). Regulation of the expression of the angiogenic enzyme platelet-derived endothelial cell growth factor/thymidine phosphorylase in endometrial isolates by ovarian steroids and cytokines. Endocrinology, 138(11), 4921-4930.

Zimmerman M. \& Seidenberg J. (1964) Deoxyribosyl transferases. Journal of Biological Chemistry, $239,2618-2621$. 